Pacific Journal of Mathematics

ON CERTAIN PROJECTIONS IN SPACES OF CONTINUOUS 


\title{
ON CERTAIN PROJECTIONS IN SPACES OF CONTINUOUS FUNCTIONS
}

\author{
S. P. LLOYD
}

1. Introduction. Let $X$ be a compact Hausdorff space and let $C(X)$ be the Banach space of continuous real or complex valued functions on $X$, with supremum norm. We are concerned with the set $\mathscr{P}$ of positive bounded constant decreasing projections in $C(X)$. That is, $\mathscr{P}$ is the set of bounded linear operators $T: C(X) \rightarrow C(X)$ which have the properties $T^{2}=T, T f \geqq 0$ if $f \geqq 0, T 1 \leqq 1$. A great deal is known about the structure of such $T$ when the range of $T$ is a closed self-adjoint subalgebra of $C(X)$ containing constants [1] [4] [5]. In the present paper we develop a corresponding representation theory for members of $\mathscr{P}$. An application to Markov processes is given.

2. Representation theory. Let $\boldsymbol{X}$ denote the $\sigma$-field of Borel subsets of $X$. We represent the conjugate space of bounded linear functionals on $C(X)$ as the space of regular real or complex Borel measures in $X$, with variation norm. In all that follows, the topology in $C^{*}(X)$ will be the $C(X)$ (weak*) topology.

THEOREM 1. The members of $\mathscr{P}$ correspond $1-1$ to certain $C^{*}(X)$ valued functions on $X$, as follows. Suppose $t: X \rightarrow C^{*}(X)$ corresponds to $T \in \mathscr{P}$. Then $t$ and $T$ are related by (i), and $t$ has properties (ii)-(iv):

(i ) $T f(x)=\int f\left(x^{\prime}\right) t_{x}\left(d x^{\prime}\right), x \in X, f \in C(X)$

(ii) $t: X \rightarrow C^{*}(X)$ is continuous (with the $C(X)$ topology in $C^{*}(X)$ ).

(iii) $t_{x} \geqq 0, t_{x}(X) \leqq 1, x \in X$

(iv) $t_{x}=\int t_{x^{\prime}} t_{x}\left(d x^{\prime}\right), x \in X$.

Proof. Suppose $T \in \mathscr{P}$ is given. Standard representation theory for bounded linear transformations into $C(X)$ gives (i) and (ii) immediately [2, p. 490]. Property (iii) is a consequence of $T \geqq 0, T 1 \leqq 1$. It is to be noted that the conditions $T \geqq 0, T 1 \leqq 1, T \neq 0$ which characterize the nonzero members of $\mathscr{P}$ are equivalent to the conditions $T \geqq 0,\|T\|=1$. The function $t$ is simply the restriction of the adjoint $T^{*}: C^{*}(X) \rightarrow C^{*}(X)$ to domain $X$, regarding $X$ as the set of unit point measures in $C^{*}(X)$. The adjoint itself has the representation

Received July 30, 1962. 


$$
T^{*} \lambda=\int t_{x} \lambda(d x), \lambda \in C^{*}(X),
$$

where the integration is in the weak* sense [3]. (That is, for given $\lambda \in C^{*}(X)$ the value of the integral in (2) is the element of $C^{*}(X)$ whose values for $f \in C(X)$ are

$$
\int \lambda(d x) \int f\left(x^{\prime}\right) t_{x}\left(d x^{\prime}\right), f \in C(X) .
$$

Condition (iv) is a consequence of $T^{2}=T$; the integration again is in the weak* sense. Conversely, any $t$ with properties (ii)-(iv) determines a $T \in \mathscr{P}$ according to (i), and the theorem is proved.

Let $\varphi$ be the equivalence in $X$ defined by $x_{1} \varphi x_{2}$ if and only if $t_{x_{1}}=t_{x_{2}}$. On the quotient space $Y=X / \varphi$ define $\bar{t}: Y \rightarrow C^{*}(X)$ by $\bar{t}_{y}=t_{x}$ if $y=\pi x, x \in X$ where $\pi: X \rightarrow Y$ is the quotient mapping. General considerations show that $\bar{t}$ is a homeomorphism of compact Hausdorff $Y$ and the set $K=\left\{\bar{t}_{y}: y \in Y\right\}$ of various distinct values of $t$. The quotient mapping is closed, so that the decomposition $\left\{\pi^{-1} y\right.$ : $y \in Y\}$ of $X$ into closed equivalence classes is upper semicontinuous.

Denote by $K_{1}$ the closed convex hull of $K \cup\{0\}$, where 0 is the zero measure. Since $K \cup\{0\}$ is compact, $K_{1}$ is compact, and is hence the closed convex hull of its extreme points. Denote by $Y_{0}$ the set of all $y \in Y$ such that $\bar{t}_{y} \neq 0$ is an extreme point of $K_{1}$; all extreme points of $K_{1}$ are to be found in $\left\{\bar{t}_{y}: y \in Y_{0}\right\} \cup\{0\}$ [2, p. 440].

THEOREM 2. For each $y \in Y_{0}$ the measure $\bar{t}_{y}$ lives on $\pi^{-1} y$; that is, $\bar{t}_{y}(E)=\bar{t}_{y}\left(E \cap \pi^{-1} y\right), E \in X, y \in Y_{0}$. Moreover, $\bar{t}_{y}(X)=1, y \in Y_{0}$.

Proof. Property (1.iv) is

$$
\bar{t}_{y}=\int \bar{t}_{\pi x} \bar{t}_{y}(d x), y \in Y,
$$

in terms of $\bar{t}$. Fix $y \in Y_{0}$, and suppose there exists a closed set $F$ disjoint from $\pi^{-1} y$ such that $\bar{t}_{y}(F)>0$. Since $\bar{t}$ is one-to-one and continuous, $\bar{t} \pi F=\left\{\bar{t}_{\pi x}: x \in F\right\}$ is a compact set which does not contain $\bar{t}_{y}$. The closed convex hull of $\bar{t} \pi F$ does not contain $\bar{t}_{y}$, either (otherwise $\bar{t}_{y} \in \bar{t} \pi F, \bar{t}_{y}$ being extreme [2, p. 440]). Thus there exists $f \in C(X)$ which separates $\bar{t}_{y}$ and $\bar{t} \pi F$ strictly. Expressing (3) as

$$
\bar{t}_{v}=\bar{t}_{y}(F) \int_{F} \bar{t}_{\pi x} \frac{\bar{t}_{y}(d x)}{\bar{t}_{y}(F)}+\int_{X-F} \bar{t}_{\pi x} \bar{t}_{y}(d x)+\left[1-\bar{t}_{y}(X)\right] 0,
$$

we see that $\bar{t}_{y}$ is expressed as a proper convex combination of elements of $K_{1}$ distinct from $\bar{t}_{y}$. This contradicts the assumption that $\bar{t}_{y}$ is an extreme point of $K_{1}$. The regularity of each $\bar{t}_{y}$ shows that $\bar{t}_{y}$ lives 
on $\pi^{-1} y$ when $y \in Y_{0}$. The same sort of argument shows that if $\bar{t}_{y}(X) \neq$ 0 , then $\bar{t}_{y}$ is not an extreme point of $K_{1}$ unless $\bar{t}_{y}(X)=1$.

THeOREM 3. $Y_{0}$ is closed.

Proof. Define $u: Y \rightarrow C^{*}(Y)$ by $u_{y}(E)=\bar{t}_{y}\left(\pi^{-1} E\right), E \in \boldsymbol{Y}, y \in Y$. The continuity of $\bar{t}$ implies that $u$ is continuous with the $C(Y)$ topology in $C^{*}(Y)$. From Theorem $2, u_{y}$ is for each $y \in Y_{0}$ the unit point measure at $y$. Thus for each $f \in C(Y)$ we have

$$
f(y)=\int f\left(y^{\prime}\right) u_{y}\left(d y^{\prime}\right), y \in Y_{0} .
$$

Since for each $f \in C(Y)$ the members of (4) are continuous in $y$, the equality (4) persists for $y \in \bar{Y}_{0}$. This implies that $u_{y}$ is the unit point measure at $y$ for each $y \in \bar{Y}_{0}$. It follows that $\bar{t}_{y}$ lives on $\pi^{-1} y$ for each $y \in \bar{Y}_{0}$. It should be clear that each such $\bar{t}_{y}, y \in \bar{Y}_{0}$, is necessarily an extreme point of $K_{1}$, and the theorem follows.

THeOREM 4. For each $y \in Y$ the measure $\bar{t}_{y}$ lives on $\pi^{-1} Y_{0}$; that is, $\bar{t}_{y}(E)=\bar{t}_{y}\left(E \cap \pi^{-1} Y_{0}\right), E \in \boldsymbol{X}, y \in Y$.

Proof. Since $\left\{\bar{t}_{y}, y \in Y\right\}$ is in the closed convex hull of compact $\left\{\bar{t}_{y}, y \in Y_{0}\right\} \cup\{0\}$, for each $y \in Y$ there exists a Borel measure $\nu_{y} \geqq 0$ on compact $Y_{0}$ such that

$$
\bar{t}_{y}=\int_{Y_{0}} \bar{t}_{y^{\prime}} \nu_{y}\left(d y^{\prime}\right)
$$

in the weak ${ }^{*}$ sense. Let $F$ be an arbitrary closed subset of $X-\pi^{-1} Y_{0}$, and let $f \in C(X)$ satisfy $f(F)=1, f\left(\pi^{-1} Y_{0}\right)=0,0 \leqq f \leqq 1$. From (5) and Theorem 2 one has $\int f(x) \bar{t}_{y}(d x)=0, y \in Y$, and hence $\bar{t}_{y}(F)=0$, $y \in Y$. Since each $\bar{t}_{y}$ is regular, the theorem follows.

3. Invariant measures and functions. We now characterize the ranges of $T^{*}$ and $T$. From (2), any invariant measure $T^{*} \lambda$ is contained in the weak ${ }^{*}$ closed subspace spanned by $\left\{t_{x}, x \in X\right\}$. From (1.iv), each $t_{x}$ is invariant, $x \in X$. Thus range $\left(T^{*}\right)$ is the weak ${ }^{*}$ closed subspace spanned by $\left\{t_{x}, x \in X\right\}$. The extreme points $\left\{\bar{t}_{y}, y \in Y_{0}\right\}$ constitute a minimal spanning set, clearly.

From (1.i), any invariant function $T f$ is constant on equivalence classes, and so determines an element of $C(Y)$. Restriction of domain to $Y_{0}$ gives an element of $C\left(Y_{0}\right)$. Conversely, let $f_{0}$ be an arbitrary element of $C\left(Y_{0}\right)$. Define function $f$ by

$$
f(x)=\int_{\pi^{-1} Y_{0}} f_{0}\left(\pi x^{\prime}\right) t_{x}\left(d x^{\prime}\right), x \in X .
$$


It follows from Theorem 3 and the Tietze extension theorem that $f \in C(X)$ and hence that $T f=f$. From Theorem 2, the contraction procedure described above applied to $f$ gives $f_{0}$ back again. It should then be clear that (6) establishes an isometric order isomorphism of $C\left(Y_{0}\right)$ and range $(T)$. The isomorphism is algebraic if and only if $Y_{0}=Y[4]$.

4. Application to Markov chains. Let $\left(X_{1}, \mathscr{F}\right)$ be a measurable space, and let $p(x, E), x \in X_{1}, E \in \mathscr{F}$, be a transition subprobability. That is, $p(x, \cdot)$ is a measure on $\mathscr{F}$ for each $x \in X_{1}$ and $0 \leqq p(\cdot, E) \leqq 1$ is a measurable function for each $E \in \mathscr{F}$. Denote by $B\left(X_{1}, \mathscr{F}\right)$ the Banach space of all bounded real or complex measurable functions on $X_{1}$, with supremum norm. Then $P: B\left(X_{1}, \mathscr{F}\right) \rightarrow B\left(X_{1}, \mathscr{F}\right)$ defined by

$$
P f(x)=\int f\left(x^{\prime}\right) p\left(x, d x^{\prime}\right), x \in X_{1}, f \in B\left(X_{1}, \mathscr{F}\right),
$$

has the properties $P \geqq 0,\|P\| \leqq 1$. Suppose there is an operator $T$ (necessarily unique) in the closed convex hull of $\left\{P^{n}, n=1,2, \cdots\right\}$ in the weak operator topology with the properties $T P=P T=T$. Then $T$ has the properties $T \geqq 0,\|T\| \leqq 1$, and is the projection onto the subspace of invariant functions of $P$.

We assume without essential loss of generality that $B\left(X_{1}, \mathscr{F}\right)$ separates the points of $X_{1}$. Then there is a totally disconnected compact Hausdorff space $X$ containing $X_{1}$ as a dense subset such that each element of $B\left(X_{1}, \mathscr{F}\right)$ extends uniquely to an element of $C(X)[2, \mathrm{p}$. 276]. Operator $P$ becomes an operator $P: C(X) \rightarrow C(X)$ with the properties $P \geqq 0,\|P\| \leqq 1$. Such an operator necessarily has the form

$$
P f(x)=\int f\left(x^{\prime}\right) p_{x}\left(d x^{\prime}\right), x \in X, f \in C(X),
$$

where $p: X \rightarrow C^{*}(X)$ is continuous with the $C(X)$ topology in $C^{*}(X)$ and has the properties $p_{x} \geqq 0, p_{x}(X) \leqq 1, x \in X$. Clearly, $p$ is the extension of the given transition subprobability to all of $X$.

The operator $T$ becomes a projection in $C(X)$ to which our results apply. Each set $\pi^{-1} y, y \in Y_{0}$ is an ergodic set and $X-\pi^{-1} Y_{0}$ is the dissipative set, according to

THEOREM 5. If $y \in Y_{0}$ then for almost all $x \in \pi^{-1} y$ with respect to $\bar{t}_{y}$ the measure $p_{x}$ lives on $\pi^{-1} y$.

Proof. From $T P=T$ we obtain

$$
\int \bar{t}_{y}(d x) \int p_{x}\left(d x^{\prime}\right) f\left(d x^{\prime}\right)=\int \bar{t}_{y}\left(x^{\prime}\right) f\left(x^{\prime}\right), y \in Y, f \in C(X) \text {. }
$$


Fix $y \in Y_{0}$ and let $F$ be any closed set disjoint from $\pi^{-1} y$. Let $f \in C(X)$ be such that $f(F)=1, f\left(\pi^{-1} y\right)=0,0 \leqq f \leqq 1$. The right-hand side above vanishes, from Theorem 2 , which requires $p_{x}(F)=0$ for almost all $x$ with respect to $\bar{t}_{y}$. Since $p_{x}$ is regular, the theorem follows.

\section{REFERENCES}

1. G. Birkhoff, Moyennes de fonctions bornées, Algèbre et Thèorie des Nombres, Colloques Internationaux du Centre National de la Recherche Scientifique, No. 24, pp. 143-153, Centre National de la Recherche Scientifique, Paris, 1950.

2. N. Dunford and J. T. Schwartz, Linear Operators I, Interscience Publishers, Inc., New York, 1958.

3. - Uniformity in linear spaces, Trans, Amer. Math. Soc., 44 (1938), 305-356.

4. J. L. Kelley, Averaging Operators on $C_{\infty}(X)$, Illinois J. Math., 2 (1958), 214-223.

5. F. B. Wright, Generalized means, Trans. Amer. Math. Soc., 98 (1961), 187-203.

Bell Telephone laboratories, InCORporated

Murray Hill, New Jersey 



\section{PACIFIC JOURNAL OF MATHEMATICS}

\section{EDITORS}

\section{RalPh S. Phillips}

Stanford University

Stanford, California

M. G. Arsove

University of Washington

Seattle 5, Washington
J. Dugundji

University of Southern California Los Angeles 7, California

Lowell J. Paige

University of California

Los Angeles 24, California

\section{ASSOCIATE EDITORS}
E. F. BECKENBACH
D. DERRY
H. L. ROYDEN
E. G. STRAUS
T. M. CHERRY
M. OHTSUKA
E. SPANIER
F. WOLF

\section{SUPPORTING INSTITUTIONS}

\author{
UNIVERSITY OF BRITISH COLUMBIA \\ CALIFORNIA INSTITUTE OF TECHNOLOGY \\ UNIVERSITY OF CALIFORNIA \\ MONTANA STATE UNIVERSITY \\ UNIVERSITY OF NEVADA \\ NEW MEXICO STATE UNIVERSITY \\ OREGON STATE UNIVERSITY \\ UNIVERSITY OF OREGON \\ OSAKA UNIVERSITY \\ UNIVERSITY OF SOUTHERN CALIFORNIA
}

STANFORD UNIVERSITY

UNIVERSITY OF TOKYO

UNIVERSITY OF UTAH

WASHINGTON STATE UNIVERSITY

UNIVERSITY OF WASHINGTON

AMERICAN MATHEMATICAL SOCIETY

CALIFORNIA RESEARCH CORPORATION SPACE TECHNOLOGY LABORATORIES

NAVAL ORDNANCE TEST STATION

Printed in Japan by International Academic Printing Co., Ltd., Tokyo Japan 


\section{Pacific Journal of Mathematics}

\section{Vol. 13, No. 1 \\ March, 1963}

Frantz Woodrow Ashley, Jr., A cone of super-(L) functions............. 1

Earl Robert Berkson, Some metrics on the subspaces of a Banach space....

Felix Earl Browder and Walter Strauss, Scattering for non-linear wave

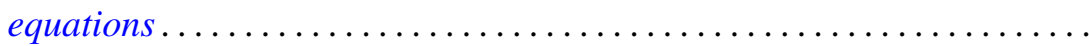

Edmond Darrell Cashwell and C. J. Everett, Formal power series ..........

Frank Sydney Cater, Continuous linear functionals on certain topological

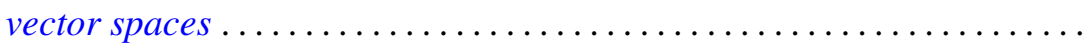

John Douglas Dixon, General group extensions ....................

Robert Pertsch Gilbert, On harmonic functions of four variables with

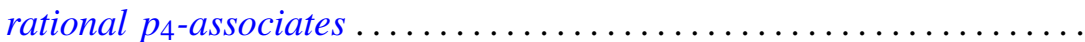

Irving Leonard Glicksberg, On convex hulls of translates ..............

Simon Hellerstein, On a class of meromorphic functions with deficient zeros

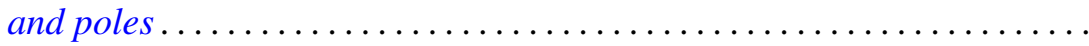

Donald William Kahn, Secondary cohomology operations which extend the

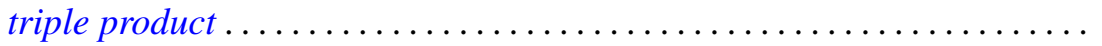

G. K. Leaf, A spectral theory for a class of linear operators .............

R. Sherman Lehman, Algebraic properties of the composition of solutions of partial differential equations ........................... 157

Joseph Lehner, On the generation of discontinuous groups ............. 169

S. P. Lloyd, On certain projections in spaces of continuous functions ...... 171 Fumi-Yuki Maeda, Generalized spectral operators on locally convex spaces ..................................

Donald Vern Meyer, $E^{3}$ modulo a 3-cell

William H. Mills, An application of linear programming to permutation groups.

Richard Scott Pierce, Centers of purity in abelian groups

Christian Pommerenke, On meromorphic starlike functions ...

Zalman Rubinstein, Analytic methods in the study of zeros of

polynomials...

B. N. Sahney, On the Nörlund summability of Fourier series

Tôru Saitô, Regular elements in an ordered semigroup . .

Lee Meyers Sonneborn, Level sets on spheres...........

Charles Andrew Swanson, Asymptotic estimates for limit point

problems .

Lucien Waelbroeck, On the analytic spectrum of Arens . .

Alvin (Murray) White, Singularities of a harmonic function of three

variables given by its series development .............

Kōichi Yamamoto, Decomposition fields of difference sets ...

Chung-Tao Yang, On the action of $\mathrm{SO}(3)$ on a cohomology manifold... 